\title{
Book rewiew : Frontières en tous genres - cloisonnement spatial et constructions identitaires
}

\author{
Patricia Zander \\ Université de Strasbourg, 3 rue de l'Argonne, 67083 Strasbourg, France \\ Correspondence: Patricia Zander (patricia.zander@unistra.fr)
}

Published: 29 November 2019

Staszak, J.-F. : Frontières en tous genres - cloisonnement spatial et constructions identitaires, Presses Universitaires de Rennes, Rennes, France, 212 pp., ISBN 978-2-7535-59035, EUE 22.00, 2017.

Voici un titre séduisant pour les étudiants comme les chercheurs en sciences sociales, car l'intérêt scientifique porté à la frontière et au genre ne se dément pas (Zajec, 2017; Cuttitta, 2015 ; Elden, 2016 [2007]).

Curieusement, cet ouvrage paru en 2017, dirigé par JeanFrançois Staszac et porté par une équipe de géographes à la pointe de ce domaine (Juliet Fall, Bernard Debarbieux...), ne connaît pas encore d'échos scientifiques notables si l'on se réfère au site Google Scholar. Il en est de même pour les recensions (élogieuses) dont il a fait l'objet, publiées sur des sites de portées européenne et transdisciplinaire (Blidon, 2017; Crézé, 2018).

Ce livre est présenté comme étant destiné « aux étudiants et étudiantes de premier cycle en sciences sociales et à toute personne intéressée par l'organisation sociale et spatiale du Monde et par ses discontinuités » (quatrième de couverture), «à ceux qui suivent un cours d'introduction à la géographie humaine, dont ils voudraient connaître les derniers développements » (p. 14). Il se dit «utile à tous les étudiants en sciences sociales qui s'intéressent à la question des identités » (p. 14). Il prolonge un cours éponyme ouvert en ligne en 2017, qui, précise l'avertissement, permet de consulter des documents complémentaires et de faire des exercices. Le chercheur pressé, aguerri ou non, en déduira sans doute trop vite qu'il s'agit donc d'un ouvrage de vulgarisation dont il peut faire l'économie de la lecture.

Les auteurs revendiquent pourtant des ambitions qui devraient attirer un large lectorat de chercheurs, érudits et curieux : une démarche innovante permettant « une autre fa- çon d'analyser la dimension spatiale des sociétés humaines » (p. 14), intégrant la géographie anglophone, grâce à une approche constructiviste et pluridisciplinaire.

Le premier chapitre théorique, mené par JeanFrançois Staszac, ouvre sur huit chapitres empiriques qui explorent une grande variété de frontières selon un plan par changement d'échelle : (2) Les frontières du genre (Jean-François Staszak); (3) Frontières urbaines et constructions identitaires (Raphaël Pieroni); (4) Frontières communautaires, ethno-régionalismes et apartheid (Frédéric Giraut); (5) Perspectives paysagères et frontières (Anne Sgard); (6) Frontières interétatiques et production d'identités collectives (Bernard Debarbieux); (7) Les grands découpages du monde (Jean-François Staszak, Juliet Fall, Frédéric Giraut); (8) L'échelle du monde : construire un monde naturel (Juliet Fall).

Le fil conducteur de l'ensemble est une question spatiale particulièrement aiguë en ce début du 21ème siècle : comment la frontière participe-t-elle à la construction de l'identité et de l'altérité des espaces? L'objectif de l'ouvrage est d'explorer « le rôle des différents types de frontières dans les constructions identitaires, [...] [car] beaucoup de groupes ne préexistent pas aux frontières qui semblent les séparer » (p. 11).

Nous choisissons de revenir particulièrement sur la démarche adoptée (premier chapitre) qui suscite d'intéressantes questions et réflexions épistémologiques. Les travaux empiriques qui s'inscrivent dans son cadre les prolongent et les diversifient. Nous en relèverons quelques-unes.

\section{Penser la frontière, penser l'espace par la frontière : la démarche et ses outils}

Cette première partie explicite la position théorique des auteurs. L'espace, l'identité, l'altérité, la frontière sont ainsi dé- 
finis et articulés pour servir la démarche constructiviste : l'espace, considéré comme un fait social à part entière (et non un simple «contenant» de la société), est à la fois produit et facteur du social : il est porteur de valeurs, hétérogène, résultant d'actions volontaires et involontaires qui obligent à le penser dans ses dimensions culturelles et politiques, voire antagonistes (décisions politiques, imaginaire et représentations... ). On notera que la recherche sur les frontières appelle souvent à dépasser des clivages disciplinaires : ici, les géographies politique et culturelle sont particulièrement mobilisées et, au-delà de la géographie, philosophes (Foucault, Butler...), sociologues et historiens (Bourdieu, Birnbaum, Goffman...) français ou anglo-saxons viennent consolider l'analyse et ses outils.

La démarche constructiviste adoptée est inséparable d'une position anti-essentialiste : la frontière et l'espace agissent l'un sur l'autre en permanence. L'identité (d'un individu ou d'un groupe) est le fruit d'actes au travers de rôles endossés, avec leurs mises en scène, qui ont pour objet d'affirmer des différences et de créer ainsi une séparation (avec d'autres individus ou d'autres groupes) : c'est «l'altérisation ».

Ainsi, la frontière n'a pas d'existence en tant que telle : elle peut être vue comme un « discours », un dispositif matériel et symbolique, qui acquiert du sens pour la société dans laquelle elle s'inscrit, en fonction de croyances partagées, d'une autorité qui ne fonctionne que parce qu'elle est acceptée. La « performativité » d'une frontière (en référence à J. L. Austin, 1962) correspond à l'adéquation entre ce qui est affirmé à son propos et la réalité : elle est autant dans la frontière ellemême et sa réalité matérielle (une ligne, un poste frontière, etc.) que dans l'énoncé des différences, des caractéristiques contrastées qui sont identifiées, acceptées, utilisées, etc. en son nom. Ces dispositifs géographiques performatifs (lieux, réalités matérielles, idées, images ... ) participent à l'imaginaire social : ils alimentent la façon de penser des individus et des groupes, leurs représentations et les modalités originales de leur appartenance. La frontière est donc une limite spécifique, qui se distingue par son rôle politique et identitaire. Elle participe à l'élaboration d'une multiplicité de dispositifs géographiques qui vont «structurer durablement le monde » (p. 29), obligeant ainsi à une lecture multiscalaire : les écoles communales en France, avec leur architecture spécifique, sont proposées en exemple d'un «nationalisme ordinaire » (p. 135); l'assignation des femmes à l'espace privé, et son corollaire, l'omniprésence masculine dans l'espace public (en particulier la nuit), l'organisation spatiale d'une salle de classe qui attribue une place à chaque individu selon son genre, relèvent d'un héritage culturel multiséculaire, développé et cultivé. Ce faisant, ces frontières sont le fruit de pouvoirs qu'elles contribuent à développer et à servir. De même, les grands découpages du monde (chap. 7) révèlent d'abord la puissance de ceux qui les énoncent et servent leurs intérêts. La frontière naît ainsi de pouvoirs aptes à s'affirmer, donc à affirmer leur identité. Indissociables, politique et identité révèlent un monde partagé en un ensemble d'aires délimitées et emboîtées, selon la métaphore du puzzle et des poupées russes.

Si les frontières correspondent autant à des cloisons mentales que physiques, les nécessaires franchissements relèvent désormais d'un «régime de franchissement» propre à chaque individu ; dépassements, contournements et « ruses » (de Certeau) - autant de leviers d'émancipation - sont toujours possibles : pour le chercheur qui, en interrogeant les frontières, remet en question certaines catégories qui ont façonné sa pensée; pour l'individu qui, en actes, remet en question le rôle qui lui est attribué et les cadres dans lesquels il doit agir. De même, envisager la nature comme un construit historiquement et spatialement situé remet en question la puissante dichotomie nature/culture; laquelle est au fondement de la naturalisation de certaines identités et de frontières qui confortent des pouvoirs au détriment d'autres (chap. 8).

Le parti pris du constructivisme, de l'analyse des pratiques et de leurs mises en scène, amènent les auteurs à utiliser une iconographie variée : cartes postales, photographies ou tableaux mettant en scène un pouvoir, une conquête de pouvoir par une transgression de frontière de l'espace, la vie quotidienne, des géosymboles (pont), des paysages..., assoient l'argumentaire par leurs analyses étayées.

\section{Frontières, identités et espaces en débat au 21ème siècle}

Ce livre est stimulant à bien des égards et nous nous contenterons de relever quelques-unes des nombreuses questions qu'il suscite. On pourrait lui reprocher une ouverture disciplinaire mesurée, pratiquée par une équipe composée (seulement) de géographes (Crézé, 2018). Mais l'exercice est difficile et on ne peut que saluer cette ouverture maîtrisée qui renforce la cohérence d'ensemble d'un texte pourtant écrit à six mains.

\subsection{Questionner les échelles et leur emboîtement}

Le parti pris de l'emboîtement des échelles est affirmé (métaphore de la poupée russe, utilisée à plusieurs reprises) mais cet emboîtement ne devrait-il pas être questionné plus nettement pour servir cette nouvelle façon d'analyser la dimension spatiale des sociétés ? L'exploration du rôle de la frontière dans la construction des identités, qui se comprend souvent sur le temps long, implique de recourir à ce schéma d'analyse efficace. Mais aujourd'hui ? Comme le signale Staszak, la notion d'échelle doit être également approchée de manière constructiviste, en laissant ouverte l'invention de niveaux qui sont fonction des acteurs et de leurs besoins (p. 34). En effet, les niveaux géographiques ne sont pas immuables et résultent de processus politiques et sociaux (Swyngedouw, 2004 ; Manson, 2008), devenant ainsi révélateurs des rapports de forces qui s'opèrent entre les acteurs. Quels niveaux d'échelles et quelles échelles deviennent dominants et viennent orienter les analyses, produire les frontières, valider 
un ordre du monde ? Marston et al. (2005) ont proposé une géographie humaine «sans échelle » car désormais fondée sur les réseaux, prenant en compte autant l'influence globale (selon eux, surreprésentée) que le niveau local. Quelles fusions de niveaux sont à l'œuvre, pour produire quels types de frontières porteuses d'identité?

\subsection{Quels acteurs aujourd'hui, pour quels projets politiques producteurs de frontières?}

Les échelles sont effectivement bouleversées par l'émergence de nouveaux acteurs, des sociétés devenues liquides (Bauman, 2013 [2005]) et des identités individuelles labiles : par exemple, les aires métropolitaines, cosmopolites, résistent à la gestion politique productrice de cloisonnement identitaire (p. 99, à propos des aires métropolitaines en Afrique du Sud et de la réforme territoriale entreprise au début des années 1990). Comment s'opèrent les ré-articulations d'échelles pour ces aires métropolitaines où se confrontent de multiples pouvoirs, à la fois produits de la mondialisation et fruits d'une trajectoire locale, porteuse d'une identité « locale»?

Comme le souligne la conclusion de l'ouvrage, les destructions/recompositions/reconstructions des identités sont à l'œuvre. Ces processus indissociables participent d'une même réalité dont peuvent s'emparer des pouvoirs aux revendications inquiétantes. Qu'en est-il des États, qui restent des figures dominantes pour produire les frontières et des identités ? L'heure ne serait plus à construire des écoles pour traduire un projet politique, marquant une différence avec l'État voisin ; le temps semblerait plutôt à l'effacement des expressions spatiales d'une démocratie ouverte sur le monde, de «la formation d'un monde commun au sens de Hannah Arendt » (Agier, $2016: 60$ ), comme le suggère l'exemple de la « Jungle de Calais » (Agier, 2016).

Car il n'est pas d'identité spatiale sans projet politique, pas de frontières sans projet politique et sans ses actes : d'où la question centrale vers laquelle semble revenir en creux chacune des analyses et des processus présentés : qui produit les projets politiques contemporains? Certains espaces semblent devenir des frontières à part entière, où se fabriquent, en réseau, de nouvelles identités - comme la ZAD de Notre-Dame des Landes (Pignocchi, 2019). Au siècle de l'image(rie), sommes-nous capables de mettre en lumière ces espaces-frontières qui parviennent à créer et nourrir de nouvelles identités spatiales grâce à leur ouverture au monde et non, seulement, leur cloisonnement?

\section{Références}

Agier, M. : Nouvelles réflexions sur le lieu des Sans-État Calais, son camp, ses migrants, Multitudes, 64, 53-61, https://doi.org/10.3917/mult.064.0053, 2016.

Austin, J. L. : How to do things with words, Oxford University Press, Oxford, 1962.

Bauman, Z. : La Vie Liquide [Liquid Life], Arthème Fayard, Paris, 2013 [2005].

Blidon, M. : Staszak J.-F. (dir.), Frontières en tous genres, Cloisonnement spatial et constructions identitaires, Rennes, PUR, Cybergeo : European Journal of Geography [En ligne], Revue de livres, p. 212, accessible à l'adresse suivante : http://journals. openedition.org/cybergeo/29079 (la date du dernier accès : 16 février 2018), 2017.

Crézé, R. : Jean-François Staszak (dir.), Frontières en tous genres, Cloisonnement spatial et constructions identitaires, Lectures [En ligne], Les comptes rendus, accessible à l'adresse suivante : http://journals.openedition.org/lectures/24357, mis en ligne le 7 mars 2018.

Cuttitta, P. : La «frontiérisation » de Lampedusa, comment se construit une frontière, L'Espace Politique [En ligne], 25|2015-1, accessible à l'adresse suivante : http://journals.openedition.org/ espacepolitique/3336, mise en ligne le 8 avril 2015.

Elden, S. : Space, knowledge and power : Foucault and geography, Ashgate Publishing, Londres, Burlington, 2016 [2007].

Manson, S. M. : Does scale exist ? An epistemological scale continuum for complex human-environment systems, Geoforum, 39, 776-788, 2008.

Marston, S. A., Jones III, J. P., and Woodward, K. : Human geography without scale, T. Inst. Brit. Geogr., 30, 416-432, 2005.

Pignocchi, A. : La recomposition des mondes, Seuil, Anthropocène, 2019.

Swyngedouw, E. : Scaled geographies : Nature, place, and the politics of scale, in : Scale and geographic inquiry : Nature, society, and method, 129-153, 2004.

Zajec, O. : Frontières - Des confins d'autrefois aux murs d'aujourd'hui, Chroniques thématiques, Paris, 2017. 